\title{
Analytical Solution of System of Volterra Integral Equations Using OHAM
}

\author{
Muhammad Akbar, ${ }^{1}$ Rashid Nawaz, ${ }^{1}$ Sumbal Ahsan, ${ }^{1}$ Dumitru Baleanu, ${ }^{2,3,4}$ \\ and Kottakkaran Sooppy Nisar $\mathbb{B D}^{5}$ \\ ${ }^{1}$ Department of Mathematics, Abdul Wali Khan University Mardan, Mardan, Khyber Pakhtunkhwa, Pakistan \\ ${ }^{2}$ Department of Mathematics, Cankaya University, Ankara 06790, Turkey \\ ${ }^{3}$ Institute of Space Sciences, Magurele-Bucharest 077125, Romania \\ ${ }^{4}$ Department of Medical Research, China Medical University Hospital, China Medical University, Taichung, Taiwan \\ ${ }^{5}$ Department of Mathematics, College of Arts and Sciences, Prince Sattam Bin Abdulaziz University, \\ Wadi Aldawaser, Saudi Arabia
}

Correspondence should be addressed to Kottakkaran Sooppy Nisar; ksnisar1@gmail.com

Received 18 September 2020; Revised 1 October 2020; Accepted 12 October 2020; Published 3 December 2020

Academic Editor: Hijaz Ahmad

Copyright (c) 2020 Muhammad Akbar et al. This is an open access article distributed under the Creative Commons Attribution License, which permits unrestricted use, distribution, and reproduction in any medium, provided the original work is properly cited.

In this work, a reliable technique is used for the solution of a system of Volterra integral equations (VIEs), called optimal homotopy asymptotic method (OHAM). The proposed technique is successfully applied for the solution of different problems, and comparison is made with the relaxed Monto Carlo method (RMCM) and hat basis function method (HBFM). The comparisons show that the present technique is more suitable and reliable for the solution of a system of VIEs. The presented technique uses auxiliary function containing auxiliary constants, which control the convergence. Moreover, OHAM does not require discretization like other numerical methods and is also free from small or large parameter.

\section{Introduction}

Differential and integral equations have their own importance and a lot of applications. These equations have been used in many fields, such as control, economics, electrical engineering, medicine, and so on, and also the system of these equations arises in modeling of different phenomena in different fields of science and technology. The exact solutions of most of the nonlinear systems are difficult to obtain; therefore, the researchers used an alternative approach to find their approximate solutions, such as the residual power series method [1], homotopy analysis method [2], wavelet-Galerkin method [3], Adomian decomposition method [4], modified reproducing kernel method [5], new homotopy perturbation method [6], Chebyshev wavelet method [7], homotopy perturbation method [8], fixed-point method [9], hat basis functions [10], modified variational iterative method [11], relaxed Monte
Carlo method [12], variational iterative method [13], collocation method [14], modified tanh-coth method [15], and generalized hypergeometric solutions [16].

In this work, OHAM is used $[17,18]$ for the solution of a system of VIEs. It is motivated by the aspiration to acquire an exciting solution of a system of VIEs by using the proposed method. The novelty of the presented technique is its flexible convergence and it provides us with a convenient way to control and adjust the approximation series. Many researchers used the proposed technique for different types of problems in literature. Iqbal et al. implemented it for Klein-Gordon equations [19] and singular Lane-Emden type equation [20], Sheikholeslami et al. used OHAM for the investigation of the laminar viscous flow [21], Hashmi et al. obtained solution of Fredholm integral equations [22], and Nawaz et al. obtained optimum solutions of fractional-order Zakharov-Kuznetsov Equations [23], three-dimensional Volterra integral equations [24], and fractional integro- 
differential equations [25]. We apply the method to obtain the approximate solution of a system of VIEs and test some numerical examples to show the effectiveness and accuracy of the method.

This paper is organized as follows. Section 2 gives the basic idea of OHAM for a system of VIEs. The application of OHAM to the system of VIEs is given in Section 3, and conclusions of the paper are given in Section 4.

\section{Basic Idea of OHAM}

In this section, we discuss the formulation of OHAM for solving the system of Volterra integral equations of the second kind following the procedure outlined in $[17,18]$. Let us consider the system of VIEs of the form

$$
W(x)=G(x)+\int_{0}^{x} K(x, s) w(s) \mathrm{d} s, \quad 0 \leq x \leq 1,
$$

where

$$
\begin{aligned}
W(x) & =\left[w_{1}(x), w_{2}(x), \ldots, w_{n}(x)\right]^{\mathrm{T}}, \\
G(x) & =\left[g_{1}(x), g_{2}(x), \ldots, g_{n}(x)\right]^{\mathrm{T}}, \\
K(x, s) & =\left[\kappa_{i j}(x, s)\right], \quad i, j=1,2, \ldots, n .
\end{aligned}
$$

In equation (1), $K(x, s)$ is the kernel, $G(x)$ are given functions, and $W(x)$ is the unknown solution of the system. Consider the $i$ th equation of (1):

$$
w_{i}(x)=g_{i}(x)+\int_{0}^{x} \sum_{j=1}^{n} \kappa_{i j}(x, s) w_{j}(s) \mathrm{d} s, \quad i=1,2, \ldots, n .
$$

First, we construct homotopy: $\psi_{i}\left(w_{i}(x, q) ; q\right)$ : $\mathfrak{R} \times[0,1] \longrightarrow \mathfrak{R}$, for equation (3), such that

$$
\psi_{i}\left(w_{i}(x, q) ; q\right)=(1-q)\left\{w_{i}(x)-g_{i}(x)\right\}=H(q)\left(w_{i}(x)-g_{i}(x)-\int_{0}^{x} \sum_{j=1}^{n} \kappa_{i j}(x, s) w_{j}(s) \mathrm{d} s\right), \quad i=1,2, \ldots, n
$$

where $H(q)=\sum_{p=1}^{m} c_{p} q^{p}$ is an auxiliary function, $c_{p}$ are auxiliary constants, $0 \leq q \leq 1$ is an embedding parameter, and $H(0)=0$.

For $q=0$, equation (4) becomes

$$
\psi_{i}\left(w_{i} ; 0\right)=w_{i}-g_{i}=0,
$$

and for $q=1$,

$$
\begin{array}{r}
\psi_{i}\left(w_{i} ; 1\right)=H(1)\left(w_{i}(x)-g_{i}(x)-\int_{0}^{x} \sum_{j=1}^{n} \kappa_{i j}(x, s) w_{j}(s) \mathrm{d} s\right) \\
i=1,2, \ldots, n
\end{array}
$$

When $q$ approaches from zero to 1 , then $w_{i}(x, 0)$ is continuously deformed to $w_{i}(x, 1)$.

For approximate solution of equation (3), using Taylor's series expansion about $q$, one can get

$$
w_{i}^{\infty}\left(x, q, c_{p}\right)=w_{i 0}(x)+\sum_{\kappa=1}^{\infty} w_{i \kappa}\left(x, c_{p}\right) q^{\kappa}, \quad i, p=1,2, \ldots, n .
$$

Substituting equation (7) into equation (4) and comparing the by comparing the coefficient of the like powers of $q$, one can get a series of problems.

$$
\begin{aligned}
& q^{0}: w_{i 0}(x)=g_{i}(x), \quad i=1,2, \ldots, n, \\
& q^{1}: w_{i 1}(x)=-c_{1}\left(\int_{0}^{x} \sum_{j=1}^{n} \kappa_{i j}(x, s) w_{j 0}(s) \mathrm{d} s\right), \quad i=1,2, \ldots, n .
\end{aligned}
$$

For $k^{\text {th }}$ order problem for $w_{\mathrm{ik}}(x), \quad i=1,2, \ldots, n$, it becomes

$$
\begin{aligned}
q^{k}: w_{i k}(x)= & w_{i k-1}(x)-c_{k}\left(\int_{0}^{x} \sum_{j=1}^{n} \kappa_{i j}(x, s) w_{j 0}(s) \mathrm{d} s\right) \\
& +\sum_{l=1}^{k-1} c_{l}\left[w_{i k-l}(x)-\int_{0}^{x} \sum_{j=1}^{n} \kappa_{i j}(x, s) w_{j k-1}(s) \mathrm{d} s\right] \\
& k=2,3, \ldots
\end{aligned}
$$

At $q=1$, equation (7) converges to the series solution:

$$
w_{i}^{\infty}\left(x, c_{p}\right)=w_{i 0}(x)+\sum_{\kappa=1}^{\infty} w_{i \kappa}\left(x, c_{p}\right), \quad i=1,2, \ldots, n .
$$

$m^{\text {th }}$ order approximation is

$$
\begin{gathered}
w_{i}^{m}\left(x, c_{p}\right)=w_{i 0}(x)+\sum_{\kappa=1}^{m} w_{i \kappa}\left(x, c_{p}\right), \\
i=1,2, \ldots, n, p=1,2,3, \ldots, m .
\end{gathered}
$$

We define the residual on substituting equation (11) into equation (3).

$$
R_{i}\left(x, c_{p}\right)=w_{i}^{m}\left(x, c_{p}\right)-g_{i}(x)-\int_{0}^{x} \sum_{j=1}^{n} \kappa_{i j}(x, s) w_{j}^{m}\left(s, c_{p}\right) \mathrm{d} s .
$$

To find $c_{p}, \quad p=1,2,3, \ldots$, we use the least squares method as follows: 


$$
\varphi_{i}\left(c_{p}\right)=\int_{a}^{b} R_{i}^{2}\left(x, c_{p}\right) \mathrm{d} x
$$

where limit of integration is the domain of the problem. Differentiating equation (13) with respect to the constant involved, we get

$$
\frac{\partial \varphi_{i}}{\partial c_{p}}=0, \quad i, p=1,2, \ldots
$$

One can easily obtain the values of $c_{p}$ from equation (14) for which the $m^{\text {th }}$ order approximation given in equation (11) is well determined.

\section{System of VIEs}

In this section, the consistency and reliability of OHAM are verified by some numerical problems, and the obtained results are compared with other methods in the form of tables; these tables clearly show the dominance of the proposed technique over these methods.

3.1. Problem 1. Consider the following system of VIEs [12]:

$$
\begin{aligned}
& w_{1}(x)=-\frac{1}{3}\left(x^{4}+x^{3}\right)+x^{2}+1+\int_{0}^{x}(x-s)^{3} w_{1}(s) \mathrm{d} s+\int_{0}^{x}(x-s)^{2} w_{2}(s) \mathrm{d} s \\
& w_{2}(x)=-\frac{1}{4}\left(\frac{x^{7}}{105}+x^{5}+x^{4}\right)-x^{3}+x+1+\int_{0}^{x}(x-s)^{4} w_{1}(s) \mathrm{d} s+\int_{0}^{x}(x-s)^{3} w_{2}(s) \mathrm{d} s, \quad x \in[0,1] .
\end{aligned}
$$

Equation (15) has exact solutions $w_{1}(x)=x^{2}+1$ and $w_{2}(x)=-x^{2}+x+1$.
Applying the proposed algorithm discussed in Section 2, one can get different order solutions, as follows.

Zeroth-order solution:

$$
\begin{aligned}
w_{10}(x)= & 1-\frac{1}{3} x^{2}\left(-3+x+x^{2}\right) \\
w_{20}(x)= & 1+x-x^{3}-\frac{x^{4}}{4}-\frac{x^{5}}{4}-\frac{x^{7}}{420}, \\
w_{11}(x)= & \frac{c_{1} x^{3}}{151200}\left(-50400-50400 x+720 x^{4}+405 x^{5}+x^{7}\right) \\
w_{21}(x)= & \frac{c_{1} x^{4}}{1663200}\left(-415800+x\left(-415800-3960 x^{2}+x^{3}\left(3465+1705 x+3 x^{3}\right)\right)\right) \\
w_{12}(x)= & \frac{1}{908107200} x^{3}\left(-302702400\left(c_{1}+c_{1}^{2}+c_{2}\right)-302702400\left(c_{1}+c_{1}^{2}+c_{2}\right) x+4324320\left(c_{1}+2 c_{1}^{2}+c_{2}\right) x^{4}\right. \\
& \left.+2432430\left(c_{1}+2 c_{1}^{2}+c_{2}\right) x^{5}+6006\left(c_{1}+2 c_{1}^{2}+c_{2}\right) x^{7}-7098 c_{1}^{2} x^{8}-2639 c_{1}^{2} x^{9}-3 c_{1}^{2} x^{11}\right), \\
w_{22}(x)= & \frac{1}{1297296000} x^{4}\left(-324324000\left(c_{1}+c_{1}^{2}+c_{2}\right)-324324000\left(c_{1}+c_{1}^{2}+c_{2}\right) x-3088800\left(c_{1}+c_{1}^{2}+c_{2}\right) x^{3}\right. \\
& \left.+2702700\left(c_{1}+2 c_{1}^{2}+c_{2}\right) x^{4}+1329900\left(c_{1}+2 c_{1}^{2}+c_{2}\right) x^{5}+2340\left(c_{1}+2 c_{1}^{2}+c_{2}\right) x^{7}-2925 c_{1}^{2} x^{8}-1005 c_{1}^{2} x^{9}-c_{1}^{2} x^{11}\right),
\end{aligned}
$$

$$
\begin{aligned}
w_{13}(x)= & \frac{1}{22230464256000} x^{3}\left(-7410154752000\left(c_{2}+c_{1}\left(1+c_{1}\right)^{2}+2 c_{1} c_{2}+c_{3}\right)\right. \\
& -7410154752000\left(c_{2}+c_{1}\left(1+c_{1}\right)^{2}+2 c_{1} c_{2}+c_{3}\right) x+105859353600\left(c_{2}+c_{1}+c_{1}^{2}\left(4+3 c_{1}\right)+4 c_{1} c_{2}+c_{3}\right) x^{4} \\
& +59545886400\left(c_{2}+c_{1}+c_{1}^{2}\left(4+3 c_{1}\right)+4 c_{2}\right) x^{5}+147026880\left(c_{2}+c_{1}+c_{1}^{2}\left(4+3 c_{1}\right)+4 c_{1} c_{2}+c_{3}\right) x^{7} \\
& -173759040 c_{1}\left(c_{1}\left(2+3 c_{1}\right)+2 c_{2}\right) x^{8}-64602720 c_{1}\left(c_{1}\left(2+3 c_{1}\right)+2 c_{2}\right) x^{9}-73440 c_{1}\left(c_{1}\left(2+3 c_{1}\right)+2 c_{2}\right) x^{11} \\
& \left.+68544 c_{1}^{3} x^{12}+19125 c_{1}^{3} x^{13}+13 c_{1}^{3} x^{15}\right),
\end{aligned}
$$




$$
\begin{aligned}
w_{23}(x)= & \frac{1}{46930980096000} x^{4}\left(-11732745024000\left(c_{2}+c_{1}\left(1+c_{1}\right)^{2}+2 c_{1} c_{2}+c_{3}\right)\right. \\
& -11732745024000\left(c_{2}+c_{1}\left(1+c_{1}\right)^{2}+2 c_{1} c_{2}+c_{3}\right) x-111740428800\left(c_{2}+c_{1}\left(1+c_{1}\right)^{2}+2 c_{1} c_{2}+c_{3}\right) x^{3} \\
& +97772875200\left(c_{2}+c_{1}+c_{1}^{2}\left(4+3 c_{1}\right)+4 c_{1} c_{2}+c_{3}\right) x^{4}+48110462400\left(c_{2}+c_{1}+c_{1}^{2}\left(4+3 c_{1}\right)+4 c_{1} c_{2}+c_{3}\right) x^{5} \\
& +84651840\left(c_{2}+c_{1}+c_{1}^{2}\left(4+3 c_{1}\right)+4 c_{1} c_{2}+c_{3}\right) x^{7}-105814800 c_{1}\left(c_{1}\left(2+3 c_{1}\right)+2 c_{2}\right) x^{8} \\
& \left.-36356880 c_{1}\left(c_{1}\left(2+3 c_{1}\right)+2 c_{2}\right) x^{9}-36176 c_{1}\left(c_{1}\left(2+3 c_{1}\right)+2 c_{2}\right) x^{11}+31331 c_{1}^{3} x^{12}+8227 c_{1}^{3} x^{13}+5 c_{1}^{3} x^{15}\right)
\end{aligned}
$$

Substituting equations (16)-(23) into $w_{i}=\sum_{k=0}^{3} w_{i k}$, for $i=1,2$, one can get the third-order OHAM solution. The approximate solution contains auxiliary constants; using the technique discussed in Section 2, one can get the following values of the auxiliary constants.

\begin{tabular}{cccc}
\hline & $c_{1}$ & $c_{2}$ & $c_{3}$ \\
\hline For $w_{1}(x)$ & -1.0002105730135733 & $1.62089016814963810 \times 10^{-7}$ & 0 \\
For $w_{2}(x)$ & -1.0001674339294635 & $9.10706259849821210 \times 10^{-8}$ & 0 \\
\hline
\end{tabular}

Using these constants, the third-order OHAM solution becomes

$$
\begin{aligned}
w_{1}(x) \approx & 1+x^{2}\left(1+x\left(2.586682803444161 \times 10^{-11}+2.586682803444161 \times 10^{-11} x-2.177931206041872 \times 10^{-9} x^{4}\right.\right. \\
& -1.225086304103284 \times 10^{-9} x^{5}-3.024904453268861 \times 10^{-12} x^{7}+4.942292985147856 \times 10^{-9} x^{8} \\
& +1.837519186786403 \times 10^{-9} x^{9}+2.08888122787343 \times 10^{-12} x^{11}-3.085284629176499 \times 10^{-9} x^{12} \\
& \left.\left.-8.608495059086216 \times 10^{-10} x^{13}-5.851526053235074 \times 10^{-13} x^{15}\right)\right) \\
w_{2}(x) \approx & +x-x^{3}+8.797558100309516 \times 10^{-12} x^{4}+8.797558100309516 \times 10^{-12} x^{5} \\
& +8.378634873750055 \times 10^{-14} x^{7}-5.548306011675386 \times 10^{-10} x^{8} \\
& -2.730118834996797 \times 10^{-10} x^{9}-4.803728167284256 \times 10^{-13} x^{11} \\
& +1.133324718039621 \times 10^{-9} x^{12}+3.893987492756097 \times 10^{-10} x^{13} \\
& +3.874614420651065 \times 10^{-13} x^{15}-6.67932785302346 \times 10^{-10} x^{16} \\
& -1.753880509617439 \times 10^{-10} x^{17}-1.06592956704597 \times 10^{-13} x^{19}
\end{aligned}
$$

3.2. Problem 2. Consider the following system of VIEs [10]:

$$
\begin{aligned}
& w_{1}(x)=-3 \cos (x)+3+\int_{0}^{x} \sin (x-s) w_{1}(s) \mathrm{d} s-\int_{0}^{x} \cos (x-s) w_{2}(s) \mathrm{d} s \\
& w_{2}(x)=e^{x}+\frac{1}{3} x^{x}-1-\int_{0}^{x} w_{1}(s) \mathrm{d} s-\int_{0}^{x} e^{x-s} w_{2}(s) \mathrm{d} s .
\end{aligned}
$$

The system in equation (27) has exact solutions $w_{1}(x)=$ $x^{2}$ and $w_{2}(x)=x$. Using the proposed algorithm, one can get the following third-order approximate solution and the auxiliary constants: 


\begin{tabular}{cccc}
\hline & $c_{1}$ & $c_{2}$ & $c_{3}$ \\
\hline For $w_{1}(x)$ & -0.7995466949308285 & 0.002890722834173066 & -0.001253980860449977 \\
For $w_{2}(x)$ & -0.8102088316012146 & 0.010500689249646119 & 0.0015000804520277254 \\
\hline
\end{tabular}

$w_{1}(x)=27.09222491836092+(-4.848664727375189-4.818444983672184 x) x$

$+\mathrm{e}^{x}(1.068431841695209+(0.3176884240001817-0.12778253683480223 x) x)$

$+(-28.160656760056128+x(-1.1491588724698+1.10079844163505 x)) \cos (x)$

$+(4.611703334149606+x(-10.082823921491624+(-0.03194563420870056+0.03194563420870056 x) x)) \sin (x)$,

$$
\begin{aligned}
w_{2}(x)= & 6.376428434455357+x(-4.892276735153841+x(5.424128884377468+1.9640922450512805) x) \\
& +\mathrm{e}^{x}-(11.610657907670205+x(2.2942522881388965+(0.1783715512035034-0.0886420248706046 x) x) \\
& +(5.234229473214848-4.324561216828857 x) \cos (x)+(19.533243571514003+(0.5318521492236276 \\
& -0.19944455595886038 x) x) \sin (x) .
\end{aligned}
$$

3.3. Problem 3. Consider the following system of VIEs:

$$
\begin{aligned}
& w_{1}(x)=x-\frac{x^{5}}{3}-\frac{x^{4}}{4}+\frac{x^{3}}{3}+\int_{0}^{x}\left(x^{2}-s\right) w_{1}(s) \mathrm{d} s+\int_{0}^{x}\left(x^{2}-s\right) w_{2}(s) \mathrm{d} s, \\
& w_{2}(x)=x^{2}-\frac{x^{3}}{2}-\frac{x^{4}}{3}+\int_{0}^{x} x w_{1}(s) \mathrm{d} s+\int_{0}^{x} x w_{2}(s) \mathrm{d} s, \quad x \in[0,1] .
\end{aligned}
$$

The system in equation (31) has exact solutions $w_{1}(x)=$ $x$ and $w_{2}(x)=x^{2}$. Using the proposed algorithm, one can get the following auxiliary constants and third-order approximate solution.

\begin{tabular}{cccc}
\hline & $c_{1}$ & $c_{2}$ & $c_{3}$ \\
\hline For $w_{1}(x)$ & -1.0483193039800733 & 0.000792340895704867 & -0.0000437460995528 \\
For $w_{2}(x)$ & -1.0678469758951452 & 0.002263150079957279 & -0.0002815239537518 \\
\hline
\end{tabular}

$$
\begin{aligned}
w_{1}(x)= & x\left(1 .+x^{2}-0.00007771018797754492+x(0.00005828264098329574+x(0.0003818527204535183\right. \\
& +x(0.0005069042207927407+x(-0.0008216358845389711+x(-0.001512942573949648 \\
& +x(-0.0007978851119508088+x(0.0018700147338272261+x(0.0017717351539895954 \\
& +x(0.00034944073528392453+(-0.001148881532032241-0.0005926311682586243 x) x))))))))
\end{aligned}
$$

$$
\begin{aligned}
w_{2}(x)= & x^{2}(1 .+x(0.0004504668772854076+x(0.0003003112515236363+x(-0.0008403580896928913 \\
& +x(-0.0023530026511400376+x(-0.0007914072605337691+x(0.0020684412093087273 \\
& +x(0.003660087185341051+x(0.0011269789420038446+x(-0.001273767715320215 \\
& +(-0.001792475116204039-0.0006263697932730246 x) x)))))))))
\end{aligned}
$$


TABLE 1: The exact solution and third-order OHAM solution for problem 1.

\begin{tabular}{lcccc}
\hline$x$ & Exact $w_{1}(x)$ & Exact $w_{2}(x)$ & OHAM $w_{1}(x)$ & OHAM $w_{2}(x)$ \\
\hline 0 & 1 & 1 & 1 & 1 \\
0.1 & 1.01 & 1.099 & 1.01 & 1.099 \\
0.2 & 1.04 & 1.192 & 1.04 & 1.192 \\
0.3 & 1.09 & 1.273 & 1.16 & 1.273 \\
0.4 & 1.16 & 1.336 & 1.25 & 1.336 \\
0.5 & 1.25 & 1.375 & 1.36 & 1.375 \\
0.6 & 1.36 & 1.384 & 1.49 & 1.384 \\
0.7 & 1.49 & 1.357 & 1.64 & 1.357 \\
0.8 & 1.64 & 1.288 & 1.81 & 1.288 \\
0.9 & 1.81 & 1.171 & 2 & 1 \\
1 & 2 & 1 & 2 & 1 \\
\hline
\end{tabular}

TABLE 2: The exact solution and third-order OHAM solution for problem 2.

\begin{tabular}{lcccc}
\hline$x$ & Exact $w_{1}(x)$ & Exact $w_{2}(x)$ & OHAM $w_{1}(x)$ & OHAM $w_{2}(x)$ \\
\hline 0 & 0 & 0 & $-8.88 \times 10^{-16}$ & 0 \\
0.1 & 0.01 & 0.1 & 0.010025 & 0.100038 \\
0.2 & 0.04 & 0.2 & 0.040058 & 0.200077 \\
0.3 & 0.09 & 0.3 & 0.090071 & 0.300055 \\
0.4 & 0.16 & 0.4 & 0.160071 & 0.399968 \\
0.5 & 0.25 & 0.5 & 0.250088 & 0.499851 \\
0.6 & 0.36 & 0.6 & 0.360159 & 0.599761 \\
0.7 & 0.49 & 0.7 & 0.490290 & 0.699745 \\
0.8 & 0.64 & 0.8 & 0.640481 & 0.799798 \\
0.9 & 0.81 & 0.9 & 0.810602 & 0.899817 \\
1 & 1 & 1 & 1.000450 & 0.999532 \\
\hline
\end{tabular}

TABLE 3: Comparison of absolute errors of relaxed Monte Carlo method (RMCM) [12] with $k=8, h=0.2$, and $N=100$ and third-order OHAM.

\begin{tabular}{lcccc}
\hline$x$ & RMCM $[12] w_{1}(x)$ & RMCM [12] $w_{2}(x)$ & OHAM $w_{1}(x)$ & OHAM \\
\hline 0 & 0 & 0 & 0 & 0 \\
0.1 & $3.52 \times 10^{-10}$ & $4.87 \times 10^{-11}$ & $2.79776 \times 10^{-14}$ & $2.17382 \times 10^{-13}$ \\
0.2 & $1.10 \times 10^{-8}$ & $7.55 \times 10^{-10}$ & $3.61045 \times 10^{-13}$ & $1.53211 \times 10^{-14}$ \\
0.3 & $1.47 \times 10^{-9}$ & $3.75 \times 10^{-9}$ & $1.81966 \times 10^{-12}$ & $5.15143 \times 10^{-14}$ \\
0.4 & $1.86 \times 10^{-7}$ & $4.01 \times 10^{-8}$ & $1.41989 \times 10^{-11}$ & $9.83658 \times 10^{-14}$ \\
0.5 & $6.55 \times 10^{-7}$ & $1.77 \times 10^{-7}$ & $5.23852 \times 10^{-11}$ & $1.56253 \times 10^{-12}$ \\
0.6 & $4.45 \times 10^{-7}$ & $4.33 \times 10^{-8}$ & $1.29325 \times 10^{-10}$ & $7.48757 \times 10^{-12}$ \\
0.7 & $1.38 \times 10^{-7}$ & $8.08 \times 10^{-8}$ & $2.20657 \times 10^{-10}$ & $2.25802 \times 10^{-11}$ \\
0.8 & $1.53 \times 10^{-5}$ & $6.18 \times 10^{-6}$ & $2.58731 \times 10^{-10}$ & $4.67144 \times 10^{-11}$ \\
0.9 & $6.38 \times 10^{-5}$ & $2.37 \times 10^{-5}$ & $5.19127 \times 10^{-10}$ & $6.76399 \times 10^{-11}$ \\
1 & $2.09 \times 10^{-5}$ & $2.88 \times 10^{-6}$ & $1.3096 \times 10^{-10}$ \\
\hline
\end{tabular}

TABle 4: Comparison of absolute errors of hat basis functions (HBF) [10] with $n=64$ and third-order OHAM.

\begin{tabular}{lcccc}
\hline$x$ & HBF $[10] w_{1}(x)$ & HBF $[10] w_{2}(x)$ & OHAM $w_{1}(x)$ & OHAM $w_{2}(x)$ \\
\hline 0 & 0 & 0 & $8.8817 \times 10^{-16}$ & 0 \\
0.1 & $7.700 \times 10^{-4}$ & $7.460 \times 10^{-4}$ & $2.51322 \times 10^{-5}$ & $3.75983 \times 10^{-5}$ \\
0.2 & $1.434 \times 10^{-3}$ & $1.533 \times 10^{-3}$ & $5.85541 \times 10^{-5}$ & $7.68171 \times 10^{-5}$ \\
0.3 & $2.054 \times 10^{-3}$ & $2.313 \times 10^{-3}$ & $7.12685 \times 10^{-5}$ & $5.54488 \times 10^{-5}$ \\
0.4 & $2.641 \times 10^{-3}$ & $3.085 \times 10^{-3}$ & $8.10555 \times 10^{-5}$ & $3.19823 \times 10^{-5}$ \\
0.5 & $3.103 \times 10^{-3}$ & $3.844 \times 10^{-3}$ & $1.58546 \times 10^{-4}$ & $1.48950 \times 10^{-4}$ \\
0.6 & $3.647 \times 10^{-3}$ & $4.583 \times 10^{-3}$ & $2.98618 \times 10^{-4}$ & $2.38864 \times 10^{-4}$ \\
0.7 & $4.089 \times 10^{-3}$ & $5.296 \times 10^{-3}$ & $4.81177 \times 10^{-4}$ & $2.55278 \times 10^{-4}$ \\
0.8 & $4.535 \times 10^{-3}$ & $5.970 \times 10^{-3}$ & $6.02408 \times 10^{-4}$ & $2.01705 \times 10^{-4}$ \\
0.9 & $4.998 \times 10^{-3}$ & $6.599 \times 10^{-3}$ & $4.45527 \times 10^{-4}$ & $1.82698 \times 10^{-4}$ \\
1 & $5.390 \times 10^{-3}$ & $7.170 \times 10^{-3}$ & $4.68227 \times 10^{-4}$ \\
\hline
\end{tabular}


TABLE 5: The exact solution and third-order OHAM solution for problem 3.

\begin{tabular}{lcccccc}
\hline$x$ & Exact $w_{1}(x)$ & OHAM $w_{1}(x)$ & $w_{1}(x)$ Absolute errors & Exact $w_{2}(x)$ & OHAM $w_{2}(x)$ & $w_{2}(x)$ Absolute errors \\
\hline 0. & 0 & 0 & 0. & 0 & 0 & 0 \\
0.1 & 0.1 & 0.0999999 & $6.76544 \times 10^{-8}$ & 0.01 & 0.010000 & $4.69687 \times 10^{-7}$ \\
0.2 & 0.2 & 0.200000 & $3.88365 \times 10^{-7}$ & 0.04 & 0.040003 & $3.66185 \times 10^{-6}$ \\
0.3 & 0.3 & 0.299999 & $6.09156 \times 10^{-7}$ & 0.09 & 0.090010 & $1.08757 \times 10^{-5}$ \\
0.4 & 0.4 & 0.400000 & $2.25143 \times 10^{-7}$ & 0.16 & 0.160019 & $1.93236 \times 10^{-5}$ \\
0.5 & 0.5 & 0.500002 & $2.49502 \times 10^{-6}$ & 0.25 & 0.250021 & $2.10611 \times 10^{-5}$ \\
0.6 & 0.6 & 0.600004 & $4.18880 \times 10^{-6}$ & 0.36 & 0.360008 & $8.03933 \times 10^{-6}$ \\
0.7 & 0.7 & 0.700002 & $1.61514 \times 10^{-6}$ & 0.49 & 0.489986 & $1.39203 \times 10^{-5}$ \\
0.8 & 0.8 & 0.799997 & $3.36620 \times 10^{-6}$ & 0.64 & 0.639988 & $1.22626 \times 10^{-5}$ \\
0.9 & 0.9 & 0.900002 & $2.19350 \times 10^{-6}$ & 0.81 & 0.810036 & $3.63494 \times 10^{-5}$ \\
1 & 1 & 0.999987 & $1.34560 \times 10^{-5}$ & 1 & 0.999929 & $7.10952 \times 10^{-5}$ \\
\hline
\end{tabular}
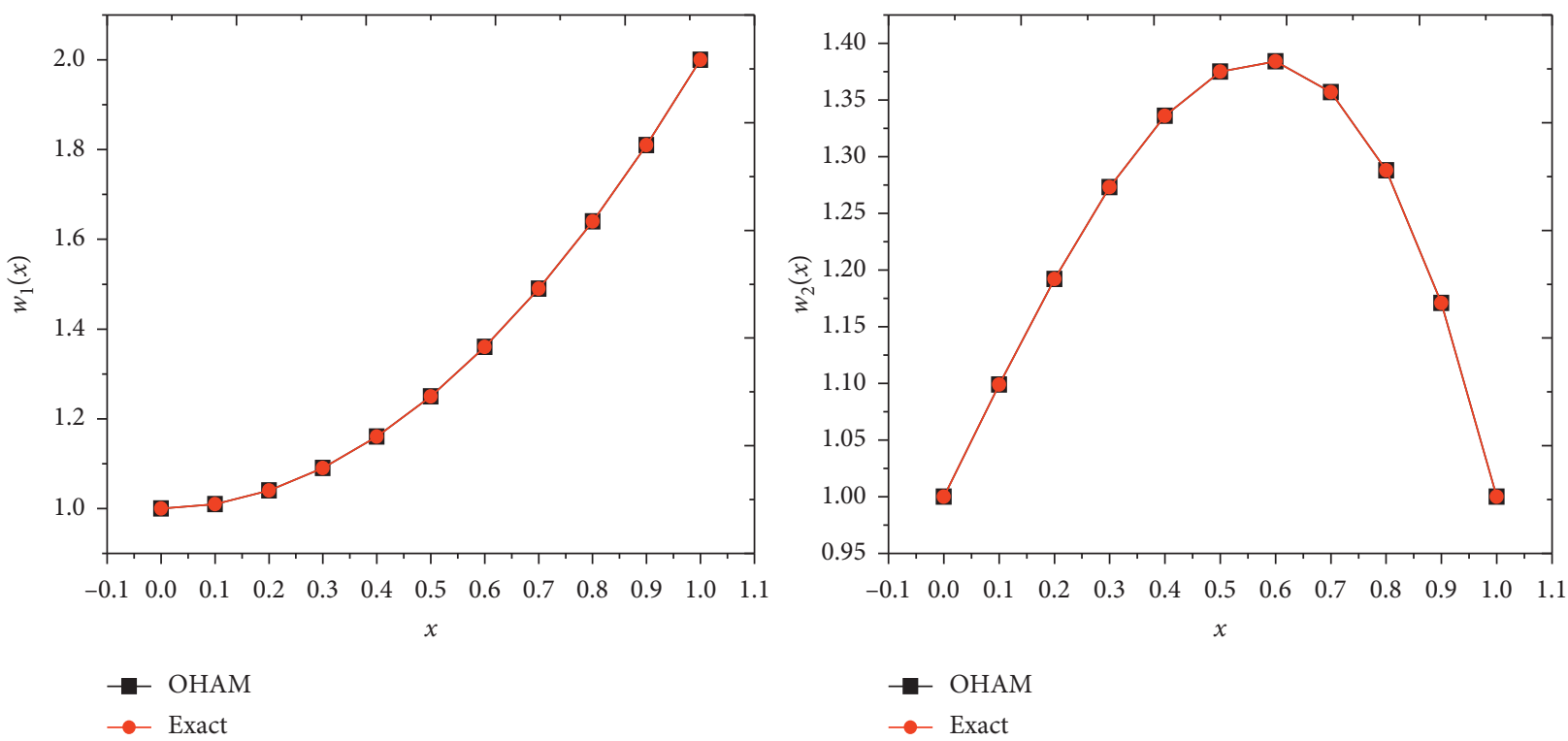

Figure 1: Plots of exact and OHAM solutions for problem 1.
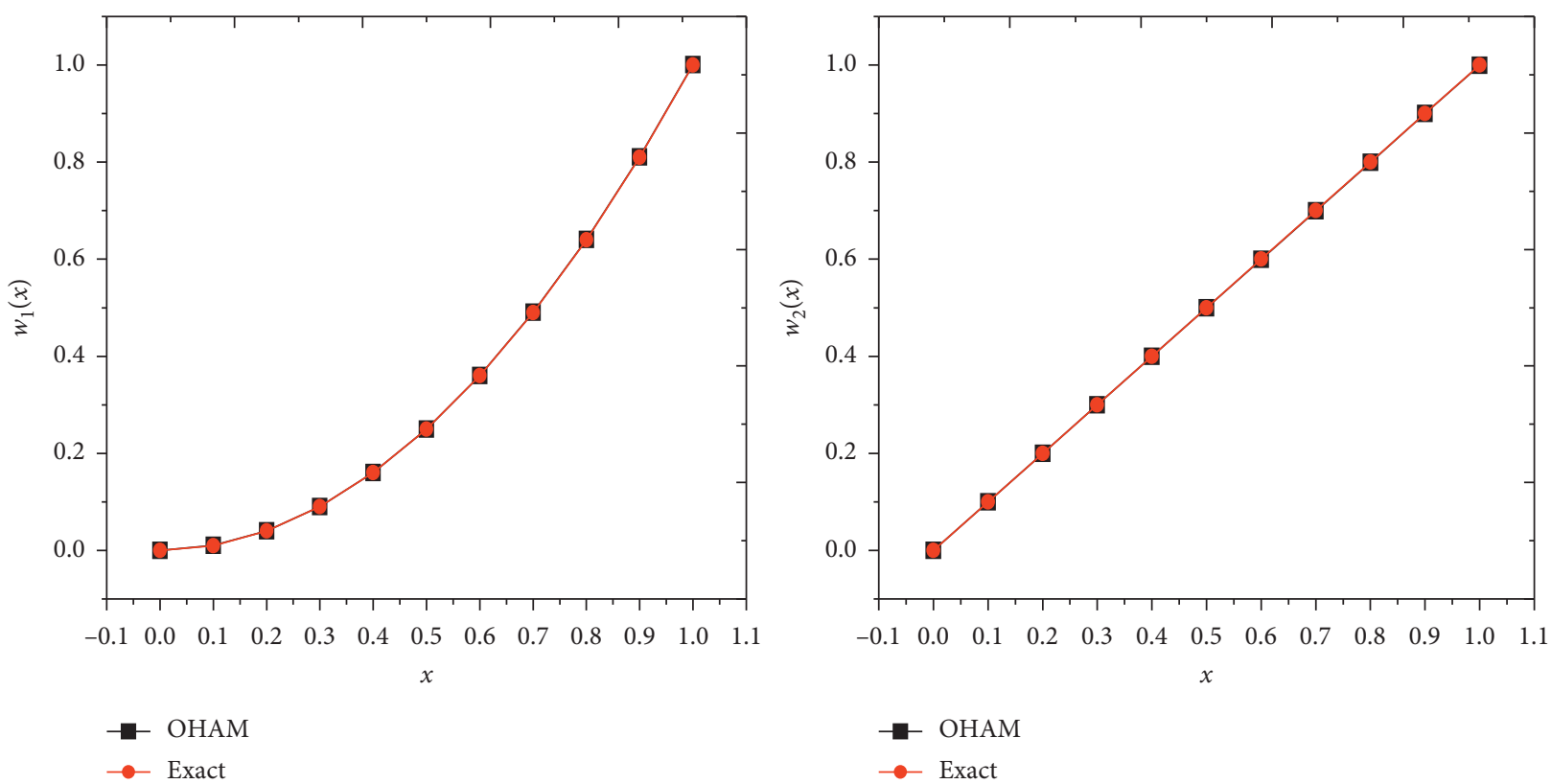

Figure 2: Plots of exact and OHAM solutions for problem 2. 

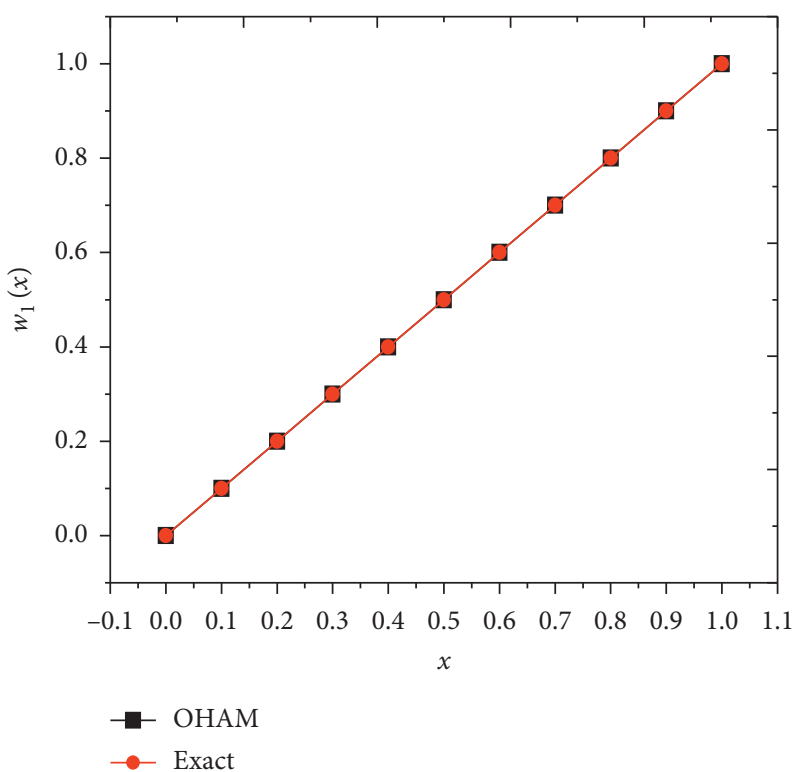

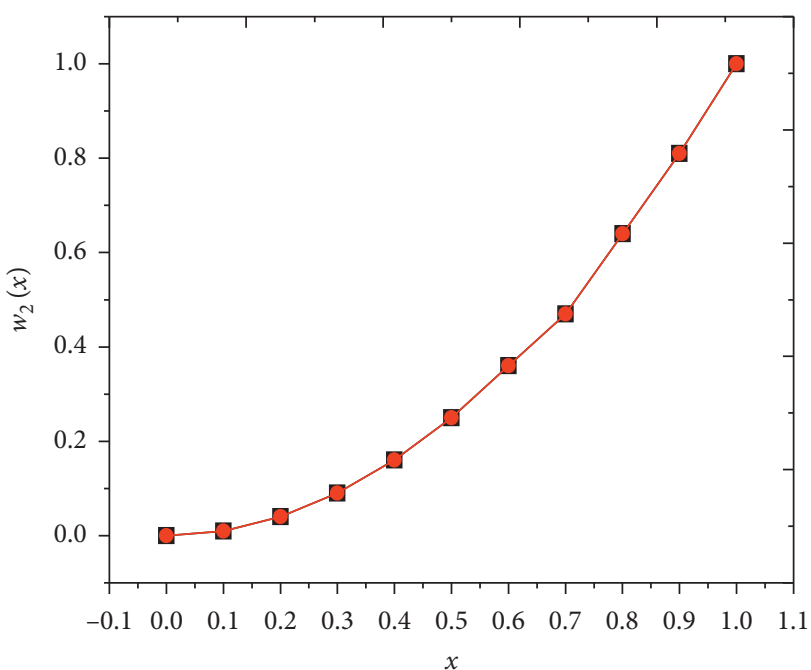

$\rightarrow-$ OHAM

$\rightarrow$ Exact

FIgURe 3: Plots of exact and OHAM solutions for problem 3.

\section{Conclusions}

Tables 1 and 2 show the exact and third-order OHAM solutions for problems 1 and 2, respectively. Third-order OHAM solutions for problem 1 are compared with the relaxed Monte Carlo method (RMCM) with $k=8, h=0.2$, and $N=100$ in Table 3, while comparison of third-order OHAM solutions is made with hat basis functions (HBF) [10] with $n=64$ in Table 4 . The OHAM solution, exact solution, and absolute errors of OHAM for problem 3 are shown in Table 5. Figures 1-3 show the plot of OHAM and exact solutions for problems 1-3, respectively. The graphical representation and tables clearly show the reliability and consistency of the proposed method for the system of VIEs.

In this paper, the simple and easy algorithm of OHAM is successfully implemented to the system of VIEs. The obtained results confirmed the reliability of the proposed method. The accuracy of the result is a point of interest; moreover, if we increase the order of approximation, the results get closer to the exact solutions. The fast convergence and accuracy of the proposed technique are valid reasons for researcher to use the OHAM for different problems arising in various fields of science and technology.

\section{Data Availability}

No data were used to support this study.

\section{Conflicts of Interest}

The authors declare that they have no conflicts of interest.

\section{References}

[1] I. Komashynska, M. Al-Smadi, A. Ateiwi, and S. Al-Obaidy, "Approximate analytical solution by residual power series method for system of fredholm integral equations," Applied
Mathematics \& Information Sciences, vol. 10, no. 3, pp. 975985, 2016

[2] A. Shidfar and A. Molabahrami, "Solving a system of integral equations by an analytic method," Mathematical and Computer Modelling, vol. 54, no. 1-2, pp. 828-835, 2011.

[3] Y. Shen and W. Lin, "A wavelet-galerkin method for a hypersingular integral equation system," Complex Variables and Elliptic Equations, vol. 52, no. 10-11, pp. 993-1006, 2007.

[4] M. S. Islam, M. S. Islam, M. Z. I. Bangalee, A. K. Khan, and A. Halder, "Approximate solution of systems of volterra integral equations of second kind by adomian decomposition method," Dhaka University Journal of Science, vol. 63, no. 1, pp. 15-18, 2007.

[5] L.-H. Yang, H.-Y. Li, and J.-R. Wang, "Solving a system of linear volterra integral equations using the modified reproducing kernel method," Abstract and Applied Analysis, vol. 2013, Article ID 196308, 2013.

[6] H. Aminikhah and J. Biazar, "A new analytical method for solving systems of volterra integral equations," International Journal of Computer Mathematics, vol. 87, no. 5, pp. 1142$1157,2010$.

[7] J. Biazar and H. Ebrahimi, "Chebyshev wavelets approach for nonlinear systems of volterra integral equations," Computers \& Mathematics with Applications, vol. 63, no. 3, pp. 608-616, 2012.

[8] J. Biazar, M. Eslami, and H. Ghazvini, "Exact solutions for systems of volterra integral equations of the first kind by homotopy perturbation method," Applied Mathematical Sciences, vol. 2, no. 54, pp. 2691-2697, 2008.

[9] T. I. Hasan, S. Salleh, and N. A. Sulaiman, "Solving a system of volterra-fredholm integral equations of the second kind via fixed point method," American Institute of Physics, vol. 1691, no. 1, pp. 1-7, 2015.

[10] E. Babolian and M. Mordad, "A numerical method for solving systems of linear and nonlinear integral equations of the second kind by hat basis functions," Computers \& Mathematics with Applications, vol. 62, no. 1, pp. 187-198, 2011.

[11] A. Armand and Z. Gouyandeh, "Numerical solution of the system of volterra integral equations of the first kind," 
International Journal of Industrial Mathematics, vol. 6, no. 1, pp. 27-35, 2014.

[12] Z. Hong, X. Fang, Z. Yan, and H. Hao, "On solving a system of volterra integral equations with relaxed Monte Carlo method," Journal of Applied Mathematics and Physics, vol. 4, no. 7, pp. 1315-1320, 2016.

[13] T. Aboiyar and H. Ibrahim, "Approximation of systems of volterra integral equations of the second kind using the new iterative method," International Journal of Applied Science and Mathematical Theory, vol. 1, no. 8, pp. 12-27, 2015.

[14] Z. Mahmoodi, "Collocation method for solving systems of fredholm and volterra integral equations," International Journal of Computer Mathematics, vol. 91, no. 8, pp. 1-16, 2014.

[15] P. Agarwal, A. A. Hyder, M. Zakarya, G. AlNemer, C. Cesarano, and D. Assante, "Exact solutions for a class of wick-type stochastic $(3+1)$-dimensional modified benjamin-bona-mahony equations," Axioms, vol. 8, no. 4, pp. 1-15, 2019.

[16] A. Ishkhanyan and C. Cesarano, "Generalized-hypergeometric solutions of the general Fuchsian linear ODE having five regular singularities," Axioms, vol. 8, no. 3, pp. 1-8, 2019.

[17] V. Marinca and N. Herişanu, "Application of optimal homotopy asymptotic method for solving nonlinear equations arising in heat transfer," International Communications in Heat and Mass Transfer, vol. 35, no. 6, pp. 710-715, 2008.

[18] N. Herişanu, V. Marinca, T. Dordea, and G. Madescu, "A new analytical approach to nonlinear vibration of an electrical machine," Proceedings of the Romanian Academy-Series A, vol. 9, no. 3, pp. 229-236, 2008.

[19] S. Iqbal, M. Idrees, A. M. Siddiqui, and A. R. Ansari, "Some solutions of the linear and nonlinear klein-gordon equations using the optimal homotopy asymptotic method," Applied Mathematics and Computation, vol. 216, no. 10, pp. 28982909, 2010.

[20] S. Iqbal and A. Javed, "Application of optimal homotopy asymptotic method for the analytic solution of singular laneemden type equation," Applied Mathematics and Computation, vol. 217, no. 19, pp. 7753-7761, 2011.

[21] M. Sheikholeslami, H. R. Ashorynejad, D. Domairry, and I. Hashim, "Investigation of the laminar viscous flow in a semi-porous channel in the presence of uniform magnetic field using optimal homotopy asymptotic method," Sains Malaysiana, vol. 41, no. 10, pp. 1281-1285, 2012.

[22] M. S. Hashmi, N. Khan, and S. Iqbal, "Optimal homotopy asymptotic method for solving nonlinear fredholm integral equations of second kind," Applied Mathematics and Computation, vol. 218, no. 22, pp. 10982-10989, 2012.

[23] R. Nawaz, L. Zada, A. Khattak, M. Jibran, and A. Khan, "Optimum solutions of fractional order zakharov-kuznetsov equations," Complexity, vol. 2019, Article ID 1741958, 2019.

[24] R. Nawaz, S. Ahsan, M. Akbar et al., "Semi analytical solutions of second type of three-dimensional volterra integral equations," International Journal of Applied and Computational Mathematics, vol. 6, no. 4, pp. 1-16, 2020.

[25] R. Nawaz, A. Khattak, M. Akbar et al., "Solution of fractionalorder integro-differential equations using optimal homotopy asymptotic method," Journal of Thermal Analysis and Calorimetry, pp. 1-13, 2020. 